\title{
Tight-Binding Method and Multiband Effective Mass Theory Applied to CdS Nanocrystals: Single-Particle Effects and Optical Spectra Fine Structure
}

\author{
J. G. Díaz, ${ }^{\dagger}$ J. Planelles, ${ }^{*}, \dagger$ G. W. Bryant, ${ }^{\ddagger}$ and J. Aizpurua ${ }^{\S}$ \\ Departament de Ciències Experimentals, Universitat Jaume I, Box 224, E-12080 Castelló, Spain, National \\ Institute of Standards and Technology, 100 Bureau Drive, Stop 8423, Gaithersburg, Maryland 20899-8423, and \\ Donostia International Physics Center, 20018, San Sebastián, Spain
}

Received: May 31, 2004; In Final Form: September 8, 2004

\begin{abstract}
Optical spectra of CdS nanocrystals are interpreted by using both the atomistic tight-binding method and multiband effective mass theory. Both methods correctly describe the energy splitting between the two lowest optically active transitions and their relative strengths, providing the same labeling of the two main absorption peaks of the spectrum. Our calculations unambiguously show that these peaks correspond to the $1 S_{3 / 2} \rightarrow 1 s$ and $1 P_{3 / 2} \rightarrow 1 p$ transitions. Both zinc blende and wurtzite-type structures for CdS nanocrystals are considered. Similar optical spectra are predicted for the two lattice structures. We also study how the spectrum, and in particular, the $1 S_{3 / 2}-1 S_{1 / 2}$ splitting, is changed by modifying parameters, within the experimental uncertainties, including size and shape fluctuations, surface passivation and spin-orbit coupling. Our results are robust to small variations in all of these parameters.
\end{abstract}

\section{Introduction}

Progress in experimental techniques allows both the synthesis and characterization of high quality, monodisperse nanocrystals with a well-established shape, crystal structure, and a consistent surface derivatization. ${ }^{1-3}$ Transmission electron microscopy (TEM) and X-ray powder diffraction (XRD) are used in combination with computer simulations to characterize nanocrystallite structural features. TEM allows imaging of individual nanocrystals and the development of a statistical description of the size and shape of the particles in a sample, whereas XRD determines the crystal structure. Different spectroscopies, such as fluorescence line narrowing and hole burning, are able to eliminate the effects of inhomogeneous broadening due to their size-selectivity so that the near-band-edge electronic fine structure can be observed. ${ }^{2}$ Recently, attenuated low-energy photoelectron spectroscopy, A-LEP, has been proposed as a new technique for studying the hole states of the quantum particles (QPs), including the splitting between the light/heavy hole and split-off bands. ${ }^{4}$

Both the effective mass method (EMM) and tight-binding (TB) theory have been successfully applied to model the optical properties of quantum dots and nanocrystals. The multiband effective mass theory has correctly predicted a large resonant Stokes shift in small CdS nanocrystals ${ }^{5}$ consistent with recent photoluminescence excitation measurements, ${ }^{6}$ whereas the atomistic tight-binding theory has provided a detailed description of the excitonic fine structure for multilayer nanocrystals with changes in composition on the monolayer scale. ${ }^{7-9}$ In the abovementioned studies, the optical properties and the various observed phenomena are described well in terms of a single isolated dot.

In this paper, we present both tight-binding and multiband effective-mass theory to describe the electronic states and optical response of CdS nanocrystals. We determine the effects of

\footnotetext{
* Corresponding author. E-mail: planelle@exp.uji.es.

$\doteqdot$ Universitat Jaume I.

$\doteqdot$ National Institute of Standards and Technology.

$\S$ Donostia International Physics Center.
}

various uncertainties that can hinder the comparison between experiment and theory. Even though current experimental protocols can reduce the nanocrystal size distribution down to $5 \%$, radius fluctuations in the lattice constant range are often still present in samples, even after applying standard size fractionation procedures. ${ }^{10}$ Thus, size variations in the nanometer range are assumed when experimental absorption spectra are obtained for samples of colloidal CdS nanocrystals of approximately $5 \mathrm{~nm} .{ }^{11}$ Morphology is another source of uncertainty because the shape of the nanocrystals is not perfectly spherical. TEM images have confirmed that $\mathrm{CdS}$ nanocrsytals exhibit a degree of ellipticity. ${ }^{12}$ The shape of the nanocrsytals has even sometimes been described in terms of thin plates rather than spheres. ${ }^{10}$ The consequences of shape uncertainty may be important because ellipticity has been predicted to affect strongly the oscillator strengths of CdSe nanocrystals ${ }^{13}$ and has been suggested as a factor that might affect the hole level splitting in CdS samples. ${ }^{4}$ Therefore, we carried out calculations for several nanocrystal sizes and shapes within the experimental uncertainty. The CdS sizes and shapes employed in our calculations correspond to those used in ref 10 and are the same as the ones assumed in the low-temperature fluorescence ${ }^{11}$ and A-LEP. ${ }^{4}$

We also assessed other possible uncertainties to check the robustness of our results. We evaluated the dependence of the low-lying hole levels on the spin-orbit parameter, $\lambda_{a}$, to see whether the surprisingly large $1 S_{3 / 2}-1 S_{1 / 2}$ splitting recently inferred from photoelectron spectroscopy ${ }^{4}$ can be explained.

The possible existence of transitions at energies below the bulk excitonic gap is addressed by modeling the presence of surface states that might arise as a consequence of an incomplete passivation of the dangling bonds by the organic ligands capping the nanocrystal (tri- $n$-octylphosphine oxide (TOPO) ${ }^{10}$ or tio-/ arachidic acids ${ }^{4}$ ). Finally, we evaluated the influence of the crystal lattice on the spectrum for the two polymorphic forms of CdS: zinc blende and wurtzite.

Our results are robust to these uncertainties. Because tightbinding is an atomistic theory, well suited for modeling small 
nanocrystals, whereas multiband effective mass theory, based on a continuum model, provides a simple labeling of the electronic states according to the global (envelope) symmetries of the nanodots, the combined use of both methods provides a good description of the near-band-edge structure in semiconductor nanocrystals.

\section{Theory and Computational Details}

2.1. Tight-Binding Theory. The tight-binding approach is an atomistic method that allows a precise, unambiguous modeling of the effects of dot size, shape, and atomic-scale variations in composition. In the TB calculations that we present here, we assume that the atoms in the dot can be located on a common regular face-centered cubic (fcc) lattice (zinc blende structure) or, alternatively, a hexagonal close packed (hcp) structure (wurtzite-type) with a two-atom basis. In our model, each atom is described by its outer valence $s$ orbital, the three outer $p$ orbitals, and a fictitious excited $s^{*}$ orbital that is included to mimic the effects of higher-lying states. The TB parameters for this basis set are taken from the zinc blende model of ref 14. By rotating the axes (to correctly describe the two different local environments for the two types of atoms in the wurtzite unit cell), we transformed the zinc blende TB parameters into the wurtzite parameters. The wurtzite bulk bands that resulted from transplanting the zinc blende parameters are in good agreement with ref 15 . Spin-orbit coupling is also incorporated in our calculations. In the TB model, the spin-orbit interaction is determined by the parameter $\lambda_{\mathrm{i}}=\left\langle x_{\mathrm{i}}, \uparrow\left|H_{\mathrm{SO}}\right| z_{\mathrm{i}}, \downarrow\right\rangle$, where $\mathrm{i}=\mathrm{a}$ (anion), $\mathrm{c}$ (cation).

In our model, coupling between atoms is limited to nearest neighbors. There are 13 different on-site and off-site parameters plus two extra parameters $\left(\lambda_{\mathrm{a}}\right.$ and $\left.\lambda_{\mathrm{c}}\right)$ that describe the spinorbit coupling. We set $\lambda_{c}=0$ because spin-orbit coupling in the conduction band is negligible. $\lambda_{a}$ is set to $30 \mathrm{meV}$ to reproduce the bulk spin-orbit splitting, unless otherwise stated. Once the nanocrystal structure was defined, the electron and hole eigenvalues were found by diagonalizing the TB Hamiltonian by means of an iterative solver. The effects of surface states in our calculations are excluded by passivating the surface dangling bonds unless otherwise specified. In our calculations, we simulate passivation by shifting the energy of each dangling bond by $V_{\mathrm{s}}=100 \mathrm{eV}$ so that dangling bonds do not modify states near the band gap.

We estimate the energies of optical transitions by the differences between the energies of the electron and hole singleparticle states, ignoring excitonic effects as they would yield, basically, a parallel shift of the spectrum. TB oscillator strengths are calculated by evaluating the dipole matrix elements using the electron and hole eigenstates. In the TB approach, dipole matrix elements are not needed to define the Hamiltonian. We estimate the dipole matrix elements between bonding orbitals on the same atom by using calculated atomic dipole matrix elements. ${ }^{16}$ The dipole matrix elements between bonding orbitals on nearest neighbor sites are chosen by reasonable guesses. The qualitative structure of the calculated spectra is insensitive to variations, within a bond length, of the bonding orbital dipole matrix elements. However, the absolute magnitude of the spectra does depend on the choice of these parameters. Because we are interested in relative strengths of different peaks, but not in absolute magnitudes, our results will not be sensitive to small variations of the selected parameters.

2.2. Multiband Effective Mass Method. We have also performed multiband calculations based on the $\mathbf{k} \cdot \mathbf{p}$ method and the envelope function approximation (EFA). We use the six-band Luttinger-Kohn Hamiltonian in the spherical ap-

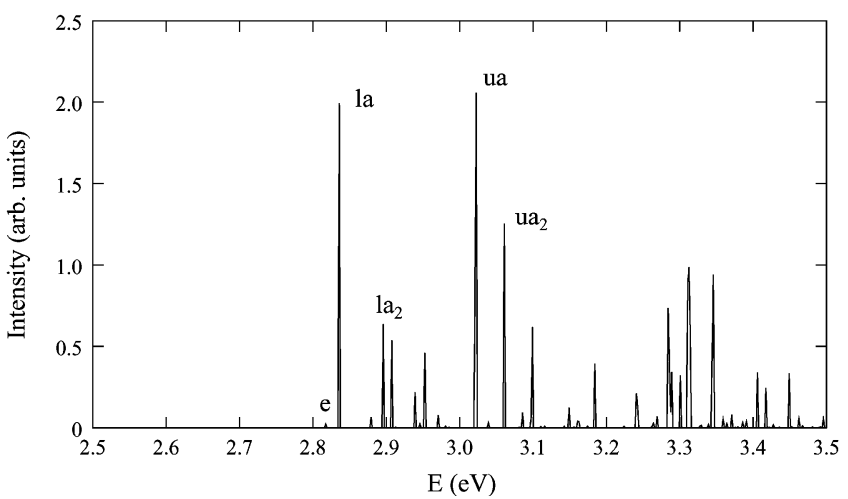

Figure 1. Near-band-edge optical spectrum for an $8 a \mathrm{CdS}$ nanocrystal. Transitions corresponding to the experimental emission $(e)$, lowest absorption $(l a)$ and first upper absorption $(u a)$ peaks are indicated. Transitions involving split-off states $1 S_{1 / 2} \rightarrow 1 s\left(l a_{2}\right)$ and $1 P_{1 / 2} \rightarrow 1 p$ $\left(u a_{2}\right)$ are also shown. The atomic dipole moments used for these calculations were $\left\langle s|x| p_{x}>=0.053 \mathrm{~nm}(\mathrm{~S}), 0.095 \mathrm{~nm}(\mathrm{Cd}) ;<s^{*}\right|$ $x \mid p_{x}>=0.032 \mathrm{~nm}(\mathrm{~S}), 0.07 \mathrm{~nm}(\mathrm{Cd})$. The dipole moment between overlapping bond orbitals from nearest neighbors $i$ and $j$ was $<i, b o \mid$ $x \mid j, b o>=0.01 \mathrm{~nm}$.

proximation to describe holes. Only the angular momentum $F$ $=J+L$, where $J$ is the Bloch band-edge angular momentum $(3 / 2$ for heavy and light holes and $1 / 2$ for split-off holes) and $L$ is the envelope angular momentum of the spherical dot, commutes with the hole Hamiltonian. Then, hole states are eigenstates of $F$ and $F_{z}$

$$
\left|F F_{\mathrm{z}} ; n Q>=\sum_{J, L \geq Q} \sum_{J_{\mathrm{z}} L_{\mathrm{z}}}<J J_{\mathrm{z}} L L_{\mathrm{z}} ; F F_{\mathrm{z}}>\right| J J_{\mathrm{z}}>\mid n L L_{\mathrm{z}}>
$$

where $\left|J J_{z}\right\rangle$ are the appropiate Bloch band-edge states, $<r \mid n L L_{\mathrm{z}}>=f_{\mathrm{nL}}(r) Y_{\mathrm{LL}}, f_{\mathrm{nL}}(r)$ are radial envelope functions, and $Y_{\mathrm{LL}_{7}}$ are spherical harmonics. We label hole states by the notation $n Q_{\mathrm{F}}$ that indicates the hole radial quantum number $n$, and the spectroscopic notation, $Q_{\mathrm{F}}$, for the lowest value of the envelope angular momentum corresponding to a given $F$. The three different radial components $f_{\mathrm{nL}}(r)$ of the envelope function are solutions of a set of second-order coupled differential equations for the radial part of the six-band Luttinger-Kohn Hamiltonian. This Hamiltonian depends on three parameters: two Luttinger parameters, $\gamma$ and $\gamma_{1}$, and the split-off coupling, $\Delta$.

Because $\mathrm{CdS}$ is a wide gap semiconductor $\left(E_{\mathrm{g}} \simeq 2.5 \mathrm{eV}\right)$, the one-band model can describe accurately the conduction band. The electron states are products of the Bloch conduction bandedge state $\mid S \sigma>$ and the envelope functions $\mid n L L_{\mathrm{z}}>$. The one-band effective mass radial equation is solved numerically to determine $f_{\mathrm{nL}}(r)$.

\section{Results}

We calculate the optical spectrum of an isolated $\mathrm{CdS}$ nanocrystal $8 a$ in diameter, where $a$ is the lattice constant ( $a=$ $0.6 \mathrm{~nm}$ ). The resulting spectrum is shown in Figure 1. Two main transitions labeled as $l a$ and $u a$ can be seen. The splitting between these two strong absorption peaks is $187 \mathrm{meV}$. Our calculations show that the hole ground state (or highest occupied molecular orbital, HOMO) is a $P$ state, in accordance with photoluminescence experiments. ${ }^{6}$ This causes a resonant Stokes shift (the energy difference between the lowest energy absorbing state and the emitting state) of $18 \mathrm{meV}$. The ground-state exciton (transition labeled as $e$ in Figure 1) that appears at $2.817 \mathrm{eV}$ is barely visible and corresponds to the $1 P_{3 / 2} \rightarrow 1 s$ transition. Tight-binding calculations show that the transition strengths are determined primarily by the spatial symmetry of the electron 


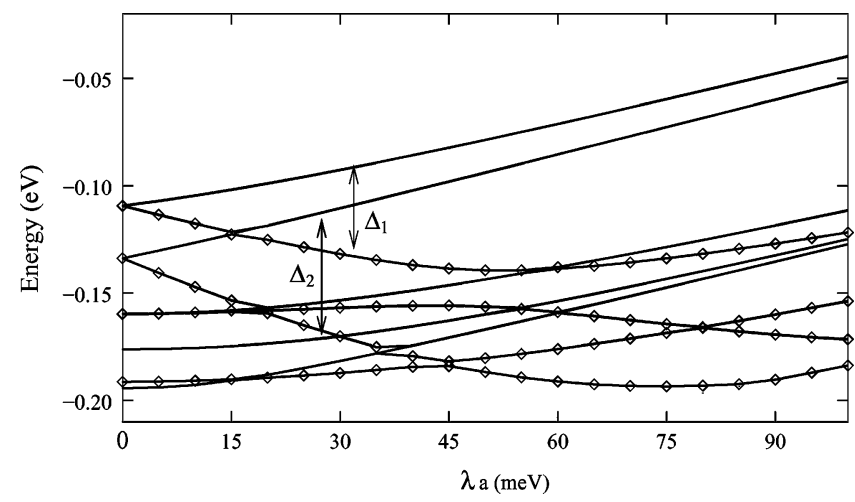

Figure 2. Dependence of the lowest hole states in an $8 a \mathrm{CdS}$ nanocrystal on the spin-orbit coupling interaction, $\lambda_{a} . \Delta_{1}$ labels the $1 P_{3 / 2}-1 P_{1 / 2}$ splitting at $\lambda_{a}=30 \mathrm{meV}$, and $\Delta_{2}$ the $1 S_{3 / 2}-1 S_{1 / 2}$ splitting at the same $\lambda_{a}$ value. Quartet states are represented by a solid line, whereas for doublets, we employ open diamonds.

and hole states. Thus, the ground state is a weakly active dim exciton because of the spatial symmetry of the states involved.

The lowest strong peak (labeled as $l a$ ) is $18 \mathrm{meV}$ Stokes shifted and corresponds to the $1 S_{3 / 2} \rightarrow 1 s$ transition, where $1 S_{3 / 2}$ is the first excited hole level. The $u a$ peak corresponds to a transition between the hole ground state, $1 P_{3 / 2}$, and the first excited electron state, $1 p$. The calculation of the oscillator strengths shows that this transition should approximately have the same intensity as the $1 S_{3 / 2} \rightarrow 1 s$, as shown in Figure 1 . The $1 S_{1 / 2} \rightarrow 1 s$ calculated transition ( $l a_{2}$ in the spectrum) appears only $60 \mathrm{meV}$ higher in energy than the lowest strong transition peak and is about three times lower than the $1 S_{3 / 2} \rightarrow 1 s$ (la) and $1 P_{3 / 2} \rightarrow 1 p(u a)$ transitions. These intensity differences come from the fact that the $1 S_{1 / 2}$ state exhibits a charge distribution displaced in the radial direction that leads to a small overlap with the $1 s$ electron state.

A-LEP spectroscopy was employed in ref 4 to study the valence band states of a matrix with six layers of CdS nanoparticles, with the same average size (5 $\mathrm{nm}$ in diameter), embedded in Langmuir-Blodgett films of variable mixtures of arachidic or thioarachidic acids. The photoelectron difference spectrum in ref 4 shows two main peaks separated by about $200 \mathrm{meV}$. Because collective properties and miniband formation appear only for highly interacting touching nanocrystals (see e.g., refs 17 and 18), the hole energy levels should be interpreted well in terms of a single dot. The observed ionization potential provides evidence that these two peaks involve intrinsic hole states of the nanoparticle and were assigned to the $1 S_{3 / 2}$ and $1 S_{1 / 2}$ depleted hole states, the splitting existing between them being related to the splitting between the $\mathrm{HH}-\mathrm{LH}$ and the splitoff bands.

The energy difference between the $1 S_{3 / 2}$ and the $1 S_{1 / 2}$ states calculated with our model just amounts to $60 \mathrm{meV}$, far from the above-mentioned $200 \mathrm{meV}$. Several reasons were given in ref 4 to justify the surprisingly large spin-orbit splitting. To check the influence of spin-orbit coupling on the lowest valence band levels, we performed calculations for different values of the spin-orbit coupling parameter $\lambda_{a}$. While changing $\lambda_{a}$, all other TB parameters were kept fixed (we can do this because bulk gaps and masses do not change much with $\lambda_{a}$ ). Figure 2 shows the dependence of low-lying hole energy states on $\lambda_{a}$. In the absence of spin-orbit coupling, the three lowest hole states are 6-fold degenerate (including the spin degeneracy). The spinorbit coupling lifts the degeneracy and produces a 4-fold degenerate state, connected with the light and heavy hole bulk bands, and a 2-fold degenerate state, the split-off band. Higher hole states can be 2-, 4-, or 6-fold degenerate. (We point out that the labeling of TB states is quite straightforward in absence of spin-orbit coupling. However, once the spin-orbit is included, there is a mixing of states and the labeling is not obvious. Energy shifts versus spin-orbit interaction strength, the oscillator strength of electron-hole transitions, and a comparison with $\mathbf{k} \cdot \mathbf{p}$ results provide us with a guide for labeling.) In bulk CdS, the zone-center splitting between the split-off band and the light and heavy hole bands is about 80 $\mathrm{meV}$. We can reproduce this splitting for bulk $\mathrm{CdS}$ by choosing $\lambda_{a}=30 \mathrm{meV}$. Therefore, we assumed this value for calculating the optical spectrum given in Figure 1 . For $\lambda_{a}=30 \mathrm{meV}$, the level splitting between $1 P_{3 / 2}$ and $1 P_{1 / 2}$, labeled by $\Delta_{1}$, is $39 \mathrm{meV}$ (this is the separation existing between the $u a$ and $u a_{2}$ transitions corresponding to the $1 P_{3 / 2} \rightarrow 1 p$ and $1 P_{1 / 2} \rightarrow 1 p$ transitions). The separation between the $1 S_{3 / 2}$ and $1 S_{1 / 2}$ has been labeled as $\Delta_{2}$ and amounts to $60 \mathrm{meV}$. Increasing $\lambda_{a}$ further separates the $1 S_{3 / 2}$ and $1 S_{1 / 2}$ states. This splitting increases linearly with $\lambda_{a}$ until $\lambda_{a} \simeq 60 \mathrm{meV}$. The slope for the $1 S_{1 / 2}$ state then decreases, flattening for higher values of $\lambda_{a}$. At $\lambda_{a} \simeq 100 \mathrm{meV}$, the slopes for the $1 S_{3 / 2}$ and $1 S_{1 / 2}$ curves are nearly the same and the splitting between both states is $132 \mathrm{meV}$, still far from $200 \mathrm{meV}$. The same qualitative behavior that is displayed in Figure 2 is obtained for a nanocrystal with a radius that is larger by one lattice constant, so size uncertainty will not change the comparison.

The low-lying hole levels (HH-LH states) shift linearly as $\lambda_{a}$ increases. However, the optical spectra obtained with higher $\lambda_{a}$ values exhibit the same general features as the spectrum at $\lambda_{a}=30 \mathrm{meV}$, that is, two strong peaks corresponding to the $1 S_{3 / 2} \rightarrow 1 s$ and $1 P_{3 / 2} \rightarrow 1 p$ transitions split about $200 \mathrm{meV}$, and a ground-state transition that remains as a dim exciton regardless of the spin-orbit coupling. By assuming $\lambda_{a}=100 \mathrm{meV}$, we can slightly reduce the Stokes shift (from 19 to $11 \mathrm{meV}$ ). The oscillator strengths corresponding to the transitions involving split-off states $1 S_{1 / 2} \rightarrow 1 s\left(l a_{2}\right)$ and $1 P_{1 / 2} \rightarrow 1 p\left(u a_{2}\right)$ decrease as $\lambda_{a}$ increases.

We also considered radius variations in the lattice constant range according to the size distributions obtained in ref 10 . The optical spectra obtained for quantum dots of sizes ranging from 42 to $54 \AA$ exhibit the same qualitative features as the spectrum in Figure 1, with the only difference being the reduction of the $u a-l a$ splitting by about $50 \mathrm{meV}$ when the radius is increased by a lattice constant.

The influence of ellipticity on the fine structure spectra has been also evaluated because TEM images show that $\mathrm{CdS}$ nanocrystals exhibit a degree of ellipticity.,12 To see how the optical spectrum is changed when oblate nanocrystals elongated in the $z$ direction are considered, we carried out calculations for nanoparticles with a varying degree of ellipticity $\left(\mu_{0}\right) . \mu_{0}$ is defined as $\mu_{0}=c_{0} / b_{0}-1$, where $c_{0}$ is the length of the long $z$ axis and $b_{0}$ is the length of the two short axes. We have considered $\mu_{0}$ values in the range of $0-0.25$ ( $b_{0}$ is kept fixed and $c_{0}$ is varied). Figure $3 \mathrm{a}$ corresponds to the absorption spectrum for a highly oblate nanocrystal $\left(\mu_{0}=0.25\right)$. The form of the spectrum is similar to the one in Figure 1, however some differences may be pointed out. On one hand, a red shift of all of the transitions is observed. The magnitude of the red shift is almost the same for all transitions. We notice slight differences only for highly oblate nanocrystals, such as those shown in Figure 3. Here, the la absorption peak is red shifted by $40 \mathrm{meV}$, a bit more than the $u a$ absorption peak $(35 \mathrm{meV})$. This stronger la shift leads to a reduction in the resonant Stokes shift, from 19 to $14 \mathrm{meV}$, and an increase in the $u a-l a$ splitting to 192 


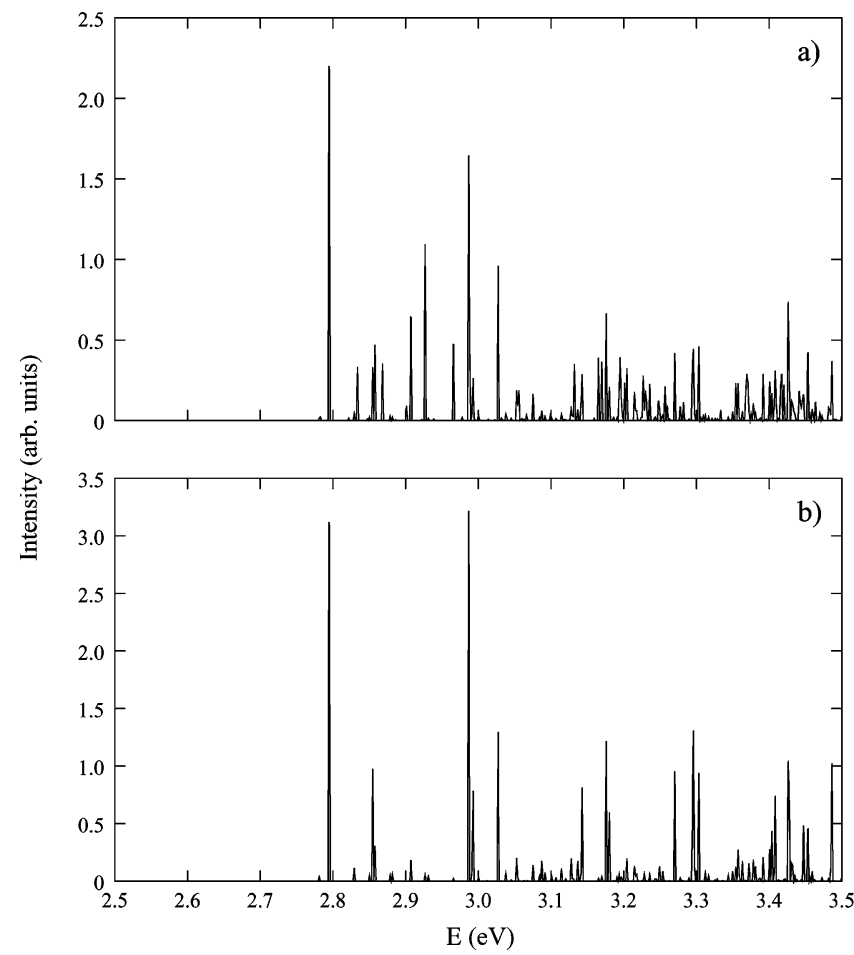

Figure 3. Optical spectra for a highly oblate $b_{0}=8 a \mathrm{CdS}$ nanocrystal $\left(\mu_{0}=0.25\right)$ using (a) nonpolarized light; (b) $z$ polarized light.

meV. On the other hand, the nanocrystal anisotropy leads to a splitting of degenerate energy levels. This can be seen in the upper part of the spectrum ( $u a$ and upward) where a sequence of low-intensity peaks appears. In particular, the crystal anisotropy lifts the degeneracy of the $1 S_{3 / 2}$ hole state, which splits into the $F_{\mathrm{z}}=1 / 2$ and $3 / 2$ components. However, the magnitude of this splitting is in the meV range and does not substantially modify the $1 S_{3 / 2}-1 S_{1 / 2}$ separation which remains about $62 \mathrm{meV}$. Thus, our calculations indicate that ellipticity cannot be responsible for the enhancement of the $1 S_{3 / 2}-1 S_{1 / 2}$ splitting, as suggested in ref 4 . Oscillator strengths in the optical spectra are also modified by changes in the morphology of the nanocrystals. In fact, the intensity of the $u a$ transition in Figure 3a decreases (although ua remains as the second strongest absorption peak) as a consequence of the difference in confinement along the $z$ direction. The $l a$ and $u a$ transitions have nearly the same intensity only for light polarized in the $z$ direction as shown in Figure $3 b$.

We try additionally to explain the existence of transitions at energies below the bulk excitonic gap observed in refs 4 and 12. In the experimentally studied samples, the nanocrystals were capped with variable mixtures of arachidic and thioarachidic acids. As shown in ref 12, the oxygen and sulfur ligands exhibit a strong tendency to bind to the Cd cation. In a previous tightbinding study of the surfaces of unreconstructed CdSe nanocrystals, ${ }^{19}$ Hill and Whaley showed that saturation with oxygen ligands very effectively removes $\mathrm{Cd}$ dangling orbitals from the band-gap region but is relatively ineffective at removing anion dangling orbitals, implying that electron traps would be removed but some hole traps would remain when capping by oxygencoordinating ligands. Therefore, we investigated whether the lack of passivation of surface $\mathrm{S}$ atoms can explain the existence of these band-gap states.

When just surface cations are passivated, an anion-derived surface-state band at about $0.5 \mathrm{eV}$ above the valence band edge exists. This band provides a series of weak, lower-energy transitions starting from $2.2 \mathrm{eV}$, which may explain the

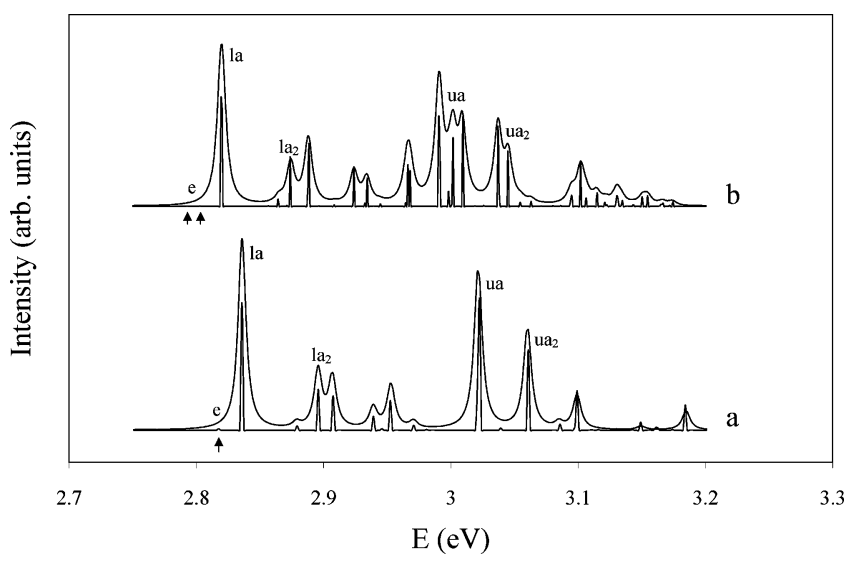

Figure 4. Near-band-edge optical spectra for a $8 a \mathrm{CdS}$ nanocrystal in the (a) zinc blende and (b) wurtzite lattice structure. Lorentzians of width $10 \mathrm{meV}$ are used to convolute the spectra. We employ the same labeling as that in Figure 1. The atomic dipole moments used for these calculations are those mentioned previously.

experimental absorption peaks at about $2.3 \mathrm{eV}$ in ref 4 . However, in the present model (only surface cations passivated), the influence of surface states on the internal electronic states destroys the form of the spectrum given in Figure 1. None of the transitions can be related to transitions of the fully passivated nanocrystal. For a better TB model of the medium surrounding the nanodot, we considered a CdS nanocrystal of radius $4 a$ with a $\mathrm{ZnS}$ layer of thickness $a$. ZnS has a larger band gap than $\mathrm{CdS}$ and is used to passivate $\mathrm{CdS}$ dangling bonds and enhance the photoluminescence of CdS. As shown in ref 20, the resulting spectrum exhibits two strong transitions split by about $200 \mathrm{meV}$, corresponding to the $1 S_{3 / 2} \rightarrow 1 s$ and $1 P_{3 / 2} \rightarrow 1 p$ transitions, that coexist with a series of weak transistions involving surface states. Thus, incomplete passivation of surface $S$ dangling bonds provides a reasonable explanation for the existence of states in the bulk band gap and is more likely than the existence of intrinsic gap states, ${ }^{21}$ as suggested in ref 4 . We find no evidence for intrinsic gap states in bare CdS.

Finally, we evaluated the effect of the crystal structure on the optical spectrum. Depending upon growth conditions, CdS crystallites can be obtained in either zinc blende or wurtzite structure. In fact, small variations in the temperature of synthesis determine which of two polymorphic forms the CdS nanocrystals will adopt. ${ }^{22}$ In Figure 4, we compare the optical spectra obtained for both lattice structures. As expected, the reduction of symmetry in the wurtzite structure increases the number of transitions. However, their splittings are not large enough to destroy the form of the spectrum obtained for the zinc blende structure. Thus, the triply degenerate electron states (6-fold if we consider the spin) corresponding to the irreducible representation $T$ convert into 1- and 2-fold degenerate states represented by the irreducible representations $A$ and $E$, respectively, for the wurtzite structure. For the $1 p$ electron states, this splitting is $7 \mathrm{meV}$, whereas the first 4-fold hole levels (considering spin), $1 P_{3 / 2}$ and $1 S_{3 / 2}$, split by 10 and $1 \mathrm{meV}$, respectively. ( Confinement leads to the discretization of the bulk bands so that the crystal field may split the resulting discrete levels in a different extension. In our case, the calculated $1 P_{3 / 2}$ crystal field splitting $(10 \mathrm{meV})$ is on the order of the experimentally determined $16 \mathrm{meV} \mathrm{HH}-\mathrm{LH}$ bulk subbands splitting, ${ }^{23}$ whereas the $1 S_{3 / 2}$ level has an almost negligible splitting $(1 \mathrm{meV})$. The discrete level splitting can be related to the anisotropy introduced by the crystal field in the set $\left(p_{x}, p_{y} p_{z}\right)$ and will depend on the wave function coefficient distribution of the different states of the corresponding multiplet.) 
The general features in the spectra that were obtained for zinc blende nanocrystals are also obtained for wurtzite nanocrystals. In fact, after convoluting the spectra with Lorentzians of $10 \mathrm{meV}$ width, the same optical structure is revealed. The separation between the la absorption band $\left(1 S_{3 / 2} \rightarrow 1 s\right.$ transitions) and the center of the band labeled as $u a\left(1 P_{3 / 2} \rightarrow 1 p\right.$ transitions) is $180 \mathrm{meV}$, and the splitting with the corresponding split-off bands $\left(l a_{2}\right.$ and $\left.u a_{2}\right)$ is approximately maintained (55 and $40 \mathrm{meV}$, respectively). The arrows in Figure 4 indicate that for a dot with a wurtzite lattice the lowest $1 P_{3 / 2} \rightarrow 1 s$ transition, which is degenerate and barely visible in a zinc blende dot, splits but remains weak. This additional splitting enhances the Stokes shift from 18 to $27 \mathrm{meV}$.

Multiband effective mass theory, based on the continuum model for the atomic lattice and $\mathbf{k} \cdot \mathbf{p}$ and envelope function approach, has also been used to model the near-band-edge fine structure. In principle, the tight-binding model is better suited than the EMM for describing small nanocrystals. Traditionally, the EMM has some difficulty reproducing energy gaps at small sizes. ${ }^{14}$ However, we have obtained the same qualitative picture when the EMM was employed. As crystal structure does not modify the general aspect of the optical spectrum, a zinc blende Hamiltonian is assumed for simplicity in the EMM calculations. The splitting between $u a$ and $l a$ transitions is the difference between the large $1 s-1 p$ electron levels separation and the much smaller Stokes shift. For the EMM calculation, we assumed a barrier of finite height $(4 \mathrm{eV})$ to model the surrounding medium. To prevent significant leakage of the electron wave function into the external medium, we assumed an electron mass $m_{\mathrm{e}}=1.0$ outside the nanocrystal, which is much larger than the CdS electron mass $\left(m_{\mathrm{e}} *=0.2\right)$ inside the dot. The $1 \mathrm{~s}-1 \mathrm{p}$ splitting calculated in the EMM approach is $219 \mathrm{meV}$, as compared to $203 \mathrm{meV}$ obtained with TB. They agree within the range of typical experimental uncertainty (30 $\mathrm{meV}$ ). The position of the $1 \mathrm{p}$ state predicted by EMM is $7 \mathrm{meV}$ lower than the one predicted by TB, whereas the $1 \mathrm{~s}$ state is about $23 \mathrm{meV}$ more bound in EMM.

$1 S_{3 / 2}$ and $1 P_{3 / 2}$ hole levels are nearly degenerate in EMM. In fact, from employing the Luttinger parameters $\gamma=0.544$ and $\gamma_{1}=2.012$ (extracted from ref 23), we found that $1 S_{3 / 2}$ is the hole ground state, in disagreement with the experimental evidence. The $1 S_{3 / 2}-1 P_{3 / 2}$ splitting obtained with such choice is $11 \mathrm{meV}$. To obtain $1 P_{3 / 2}$ as the hole ground state, we decreased the light hole/heavy hole $\left(m_{\mathrm{lh}} / m_{\mathrm{hh}}\right)$ ratio below $0.215{ }^{6}$ In the literature, we found considerable dispersion in the $\gamma$ and $\gamma_{1}$ values for the CdS nanocrystals..$^{5,23-26}$ The first two references provide energy positions closer to the ones obtained with TB. We performed calculations within the range determined by these two references, $\gamma_{1} \simeq(2.0,2.3)$ and $\gamma \simeq(0.5,0.9)$. By taking pairs of parameters that exhibit a low $m_{\mathrm{lh}} / m_{\mathrm{hh}}$ relationship, such as $\gamma=0.80$ and $\gamma_{1}=2.00$, we get a Stokes shift of 13 meV, which provides a $u a-l a$ splitting of $206 \mathrm{meV}$. We must stress at this point that the Stokes shift will only be observed if at least a six-band model (heavy and light hole and split-off bands included) is considered. In the limit of $\Delta_{S O} \rightarrow \infty$ (fourband model), the $1 S_{3 / 2}$ is always the hole ground state in the EMM (note that in the TB, even when the spin-orbit vanishes, the ground state can be either $\mathrm{S}$ or $\mathrm{P}$ depending on the surface passivation).

The magnitude of the $1 S_{3 / 2}-1 S_{1 / 2}$ splitting has also been evaluated in EMM. By assuming the parameters from ref $23(\gamma$ $=0.544, \gamma_{1}=2.012$ ), we obtained a separation of $66 \mathrm{meV}$, almost identical to the one predicted in TB. This splitting reduces when $\gamma$ is increased and amounts to $52 \mathrm{meV}$ for $\gamma=0.8$ and $\gamma_{1}=2.0$. We determined the influence of an arbitrary increase in the spin-orbit coupling parameter $\Delta$ on the above-mentioned splitting. Even employing the Luttinger parameters reported in ref 23 and a $\Delta$ value three times larger than those assumed in the literature, ${ }^{5}$ the $1 S_{3 / 2}-1 S_{1 / 2}$ splitting only reaches $110 \mathrm{meV}$.

In summary, both tight-binding and effective mass theory provide similar description for the near-band-edge electronic states and the fine structure in the optical spectra of CdS nanocrystals. This description leads us to assign the main absortion peaks in the spectra. Our calculations are robust to variations in size, shape of the nanocrystals, spin-orbit coupling, surface passivation, and lattice structure within the uncertainty of these parameters.

The magnitude of the $1 S_{3 / 2}-1 S_{1 / 2}$ splitting has also been evaluated. Both TB and EMM predict a separation significantly lower than the one suggested in ref 4 from A-LEP spectroscopy. Morphological distortion from the spherical shape of the nanocrystals, high values of the spin-orbit coupling parameter, and different dot sizes in the range of the experimental uncertainty cannot provide for such large splitting. The agreement of atomistic TB and macroscopic EMM approaches calls for new experiments to determine the origin of the doublet in the difference A-LEP spectrum.

Acknowledgment. Financial support from MEC-DGI project CTQ2004-02315/BQU and UJI-Bancaixa project P1-B2002-01 are gratefully acknowledged. Generalitat Valenciana FPI grant (JD) is also acknowledged.

\section{References and Notes}

(1) Murray, C. B.; Norris, D. J.; Bawendi, M. G. J. Am. Chem. Soc. 1993, 115,8706

(2) Mews, A.; Kadavanich, A. V.; Banin, U.; Alivisatos, A. P. Phys. Rev. B 1996, 53, R13242.

(3) Cao, Y. W.; Banin, U. J. Am. Chem. Soc. 2000, 122, 9692

(4) Samokhvalov, A.; Berfeld, M.; Lahav, M.; Naaman, R.; Rabani E. J. Phys. Chem. B 2000, 104, 8631.

(5) Li, J.; Xia, J.-B. Phys. Rev. B 2000, 62, 12613.

(6) Yu, Z.; Li, J.; O'Connor D. B.; Wang L.-W.; Barbara P. F. J. Phys. Chem. B 2003, 107, 5670.

(7) Little, R. B.; El-Sayed, M. A.; Bryant, G. W.; Burke, S. J. Chem. Phys. 2001, 114, 1813.

(8) Pérez-Conde, J.; Bhattacharjee, A. K. Phys. Rev. B 2003, 67, 235303.

(9) Bryant, G. W.; Jaskólski, W. Phys. Rev. B 2003, 67, 205320.

(10) Trindade, T.; O'Brien, P.; Zhang, X. Chem. Mater. 1997, 9, 523.

(11) Koberling, F.; Mews, A.; Basché, T. Phys. Rev. B 1999, 60, 1921.

(12) Guo, S.; Konopny, L.; Popovitz-Biro, R.; Cohen, H.; Porteanu, H.; Lifshitz, E.; Lahav, M. J. Am. Chem. Soc. 1999, 121, 9589.

(13) Albe, V.; Jouanin, C.; Bertho, D. Phys. Rev. B 1998, 58, 4713.

(14) Lippens, P. E.; Lannoo, M. Phys. Rev. B 1989, 39, 10935

(15) Wang, Y. R.; Duke, C. B. Phys. Rev. B 1988, 37, 6417.

(16) Fraga, S.; Muszynska, J. Atoms in External Fileds; Elsevier: New York, 1981

(17) Díaz, J. G.; Planelles, J.; Jaskólski, W.; Aizpurua, J.; Bryant, G W. J. Chem. Phys. 2003, 119, 7484.

(18) Díaz, J. G.; Planelles, J. Phys. Chem. B 2004, 108, 2873

(19) Hill, N. A.; Whaley, K. B. J. Chem. Phys. 1994, 100, 2831.

(20) Bryant, G. W.; Jaskólski, W. Surface States in Passivated, Unpassivated and Core/Shell Nanocrystals: Electronic Structure and Optical Properties. In Ouantum Dots, Nanoparticles and Nanowires; Proceedings of the MRS 2003 Fall Meeting, Vol. 789, Dec. 1-5, 2003; Guyot-Sionnest, P.; Mattoussi, H.; Woggon, U.; Wang, Z.-L., Eds.; Materials Research Society: Warrendale, PA, 2004.

(21) Sercel, P. C.; Efros, Al. L.; Rosen, M. Phys. Rev. Lett. 1999, 83, 2394.

(22) Kobayashi, M.; Nakamura, S.; Wakao, K., Yoshikawa, A.; Takahashi, K. J. Vac. Sci. Technol., B 1998, 36, 1317.

(23) Madelung, O., Ed. Numerical Data and Functional Relationships in Science and Technology; Springer-Verlag: Berlin, 1982.

(24) Fonoberov, V. A.; Pokatilov, E. P.; Balandin A. A. Phys. Rev. B 2002, 66, 085310

(25) Pokatilov, E. P.; Fonoberov, V. A.; Fomin, V. M.; Devreese, J. T. Phys. Rev. B 2001, 64, 245328.

(26) Efros, Al. L.; Rosen, M. Phys. Rev. B 1998, 58, 7120. 